\title{
Occurrence of paratubal cysts in childhood: An analysis of 26 cases
}

\author{
Oktav Bosnalı, Serdar Moralıoğlu, Ayşenur Cerrah-Celayir \\ Department of Pediatric Surgery, Zeynep Kamil Maternity and Children's Training and Research Hospital, Istanbul, \\ Turkey. E-mail: droktav@gmail.com \\ Received: 18 July 2016, Revised:3 August 2016, Accepted: 16 August 2016
}

SUMMARY Bosnalı O, Moralıŏlu S, Cerrah-Celayir A. Occurrence of paratubal cysts in childhood: an analysis of 26 cases. Turk J Pediatr 2016; 58: 266-270.

A retrospective analysis was conducted on the case files of patients operated on for adnexal pathology between January 2004 and December 2015 at our institution. The data of the patients with a diagnosis of paratubal cysts (PTCs) was extracted. A total of eighty-seven children were operated on for adnexal pathology. Twenty-six $(29.9 \%)$ patients had PTCs. The patients were predominantly grouped as adolescents $(n=22)$ and newborns $(n=3)$. One of the patients was a pre-pubertal girl. Of the 26 PTC cases, 18 of them occurred on the contralateral side to ovarian pathology (OP) or physiologic ovarian changes (POC) such as corpus luteum cyst or follicular cyst which their final diagnosis confirmed by histopathology examination; in one case, the PTCs were bilateral; and in three cases, PTCs were ipsilateral with OP or POC. There were only 4 solitary PTC cases identified among the 26 cases. Paratubal cysts can be observed at every age, even in newborns. Their association with OP or POC in childhood cases needs to be clarified to further understand the hormonal mechanisms that are influencing the occurrence of these cysts.

Key words: adolescent, newborn, ovary, paratubal cyst.

Paratubal cysts (PTCs) are the remnants of the congenital paramesonephric duct and are considered benign cysts ${ }^{1,2}$. They usually become symptomatic in the third or fourth decade of life with increasing size, and they can cause tubal and/or ovarian torsion, in addition to other complications ${ }^{1,3,4}$. However, they should not be confused to hydatid cyst of Morgagni. PTC's are usually located in mesosalpinx and the latter is usually located at the fimbria end of tuba uterina. The reported cases of PTCs and tubal-ovarian torsion due to the presence of PTCs in the adolescent period are rare ${ }^{4}$. Furthermore, PTCs in the neonatal period have not been reported to date. The occurrence of PTCs has been attributed to hormonal factors, but a clear association has not been demonstrated ${ }^{1,5}$. In a recent report, an association between polycystic ovary syndrome (PCOS) and PTCs was mentioned ${ }^{6}$; however, there have been no other reports suggesting a possible association between OP or a POC and PTCs.
When we retrospectively examined the clinical records of the cases operated on for adnexal pathology, we noted that PTCs frequently occurred with an OP or POC. Although most of the PTCs were asymptomatic and were found incidentally during surgical exploration for another adnexal pathology, the rate of tubalovarian torsion linked to a para-ovarian cyst was considerable. Thus, we decided to evaluate the incidence and complication rates of PTCs in children operated on for adnexal pathology in our clinic as well as the association between PTC and childhood OP or POC. In doing so, we aimed to discuss the factors contributing to PTC occurrence in childhood and to draw attention to these benign cysts.

\section{Material and Methods}

A retrospective analysis was conducted on all patients with a diagnosis of PTC. The data were extracted from the case files of patients operated on for adnexal pathology between January 2004 and December 2015 in the Department of 
Pediatric Surgery. The collected data included patient age, laboratory results, accompanying adnexal pathology, and complications caused by paratubal cysts. Informed consent was obtained from all of the patients, which included consent in obtaining the operation type and the possible complications as well as the possible publication of the results. Prior to the study, institutional review board (IRB) permission was obtained to review the clinical case records of patients with adnexal pathology (IRB No: 20/November/2015-176).

The results of our descriptive analysis are presented as the means, standard deviations, and frequencies for normally distributed variables.

\section{Results}

Eighty-seven children were operated on for adnexal pathology between January 2004 and December 2015. Of the 87 adnexal pathology patients, 26 (29.9\%) patients had PTCs which diagnosed by detailed peri-operative bilateral adnexal examination.

Of the 87 patients, 15 were diagnosed prenatally or in the newborn period as having a complicated ovarian cyst or large ovarian cyst (more than $5 \mathrm{~cm}$ in diameter) and were operated on in the newborn period. Of these 15 cases, three $(20 \%)$ of them had PTCs. Neonatal PTCs were less than $1 \mathrm{~cm}$ in diameter and were found incidentally during adnexal exploration.

There were 71 adolescent cases and one pre-pubertal case operated on for adnexal pathology. The total number of the PTC cases (either symptomatic or asymptomatic) among adolescent cases was 22 (31\%). The mean age of these 22 patients was $14.45 \pm 1.73$ years old. Of the 71 symptomatic adnexal mass cases in adolescents, $5(7 \%)$ of them were related to a paratubal cyst. Of those five cases, three of them caused adnexal torsion and related symptoms without associated OP or POC. The main symptom was colicky pain with vomiting in four cases because of tubal-ovarian torsion. In one case, a PTC was detected prior to surgery by US in a clinical setting because it caused intermittent colicky pain and the patient was admitted to the clinic. In twenty-one cases, PTCs were found incidentally during surgery for adnexal pathology.

Laboratory tests were performed, and tumor markers, including inhibin A-B, alpha fetoprotein (AFP), B-hCG, and CA-125, were studied in all adnexal mass cases. In the following three cases, the CA-125 levels were elevated: in one case with a contralateral mature cystic teratoma (41 $\mathrm{U} / \mathrm{ml})$, in one case with tubal-ovarian torsion due to a paratubal cyst $(148.6 \mathrm{U} / \mathrm{ml})$ and in one case with contralateral tubal-ovarian torsion due to a corpus luteum cyst (38.9 U/ $\mathrm{ml})$. Otherwise, all of the laboratory test results were within normal ranges.

Ultrasonography was not helpful in diagnosing PTCs in our cases preoperatively except in one case that had a large uncomplicated solitary PTC. All the remaining PTC cases either caused adnexal torsion or occurred synchronous with OP or with POC that were diagnosed during surgery.

Of the 26 PTC cases, 18 of them occurred on the contralateral side to an OP or POC. In one case, PTCs were bilateral, and in three cases, the PTCs were ipsilateral to an OP or POC. There were only 4 solitary PTC cases found. In 22 cases, OPs or POPs were detected in 23 ovarian units; however, in one case, we could not find the pathology report to indicate which OP or POC was associated with the PTC. Thirteen of the ovaries had non-neoplastic changes. Nine

Table I. Histopathologic Diagnoses of the Ovaries of the Cases in which Paratubal Cysts Occurred Concomitantly $(n=22)$

\begin{tabular}{llll}
\hline & $\mathrm{n}(\%)$ & & $\mathrm{n}(\%)$ \\
\hline Non-neoplastic & $13(59)$ & Neoplastic & $9(41)$ \\
Follicular cyst & $1(4.5)$ & Serous cystadenofibroma & $3(13.6)$ \\
Simple serous cyst & $8(36.6)$ & Serous mucinous cystadenoma & $1(4.5)$ \\
Corpus luteum cyst & $4(18.2)$ & Mature cystic teratoma & $3(13.6)$ \\
& & Disgerminoma & $1(4.5)$ \\
& & Struma ovarii & $1(4.5)$ \\
\hline
\end{tabular}


of the ovaries had neoplastic changes. Table I summarizes the histopathologic diagnoses with which PTC cases occurred concomitantly. Histopathological investigation of the PTCs showed no pre-malignant or malignant lesions in any of the extirpated cysts.

The complication rate of the PTCs that manifested as tubal-ovarian torsion was $22.2 \%$ among all of the tubal-ovarian torsion cases in our clinic. Among these cases, only three of them had solitary PTCs. In one tubal-ovarian torsion case, there was also associated ovarian pathology. Tubal-ovarian torsion was treated by salpingectomy in three cases and cyst excision with tubal salvage in one case after detorsion. In all four cases, we were able to preserve the ovaries. The side distributions and complication rates are summarized in Table II.

\section{Discussion}

The reported incidence of PTCs is approximately $10 \%$ among all adnexal pathology in adults ${ }^{2}$; however, its prevalence in healthy women is not known ${ }^{7}$. The incidence of PTCs among adolescents ranges between $4 \%$ and $7.3 \%^{1,5}$. In our study, the incidence of PTC was $7 \%$ among 71 adolescent adnexal mass cases. We could not identify the incidence of the PTCs in neonates, because none of them were the cause of primary pathology; however, the occurrence rate in neonates was lower than in adolescent cases (20\% and $32 \%$, respectively).

Paratubal cysts are embryonic remnants of tubal (paramesonephric), mesothelial and mesonephric structures. Thus, they contain tubal-type epithelium, and the cystic dilatation of PTCs is attributed to the secretory activity of the tubal-type epithelium ${ }^{1}$. It is known that menarche indicates a fully functional hypothalamic pituitary gonadal axis. Some previous papers have suggested that the occurrence of PTCs in the post-pubertal period is mediated by hormones as the tubal epithelium begins to respond to hormonal activity after puberty, thus becoming dilated ${ }^{1,6}$. Another phenomenon that suggests that hormonal activity affects PTC growth is the rapid growth of PTCs during pregnancy ${ }^{3}$. Although, androgen receptors within Wolffian remnants have been demonstrated (i.e. PTCs), no direct associations between hormonal activity and PTCs have been clearly demonstrated ${ }^{5,6}$.

According to a previous report, there is an association between PTC size and menarche, and a correlation between cyst size and body mass index also exists ${ }^{5}$. Another paper reported that hyperandrogenism seemed to be associated with larger PTCs. This same paper reported an association between obesity and PTC occurrence $^{6}$. All of the aforementioned reports suggest that different hormones affect the cystic dilatation of these embryogenic remnants and influence the occurrence of PTCs.

There were three neonatal and one pre-pubertal ovarian cyst cases with concomitant PTCs. Although PTC occurrence in pre-pubertal girls was reported, PTC occurrence in the newborn

Table II. Side Distribution and Symptomatology of Paratubal Cysts in All Cases

\begin{tabular}{|c|c|c|c|}
\hline \multirow[t]{2}{*}{ Features } & \multirow{2}{*}{$\begin{array}{l}\text { Neonatal } \\
(\mathrm{n}=3)\end{array}$} & \multirow{2}{*}{$\begin{array}{l}\text { Prepubertal } \\
(\mathrm{n}=1)\end{array}$} & \multirow{2}{*}{$\begin{array}{l}\text { Adolescent }(\mathrm{n}=22) \\
\mathrm{n}(\%)\end{array}$} \\
\hline & & & \\
\hline Asymptomatic & $3(100)$ & $1(100)$ & $17(77.3)$ \\
\hline Symptomatic & - & - & $5(22.7)$ \\
\hline Torsion & - & - & $4(18.2)$ \\
\hline \multicolumn{4}{|l|}{ Side in all PTC cases } \\
\hline Right & $1(33)$ & - & $10(45.46)$ \\
\hline Left & $2(67)$ & $1(100)$ & $9(41)$ \\
\hline Bilateral & - & - & $3(13.63)$ \\
\hline PTC side compared to the OP or POC & $(n=3)$ & $(n=1)$ & $(n=18)$ \\
\hline Contralateral & $3(100)$ & $1(100)$ & $14(77.7)$ \\
\hline Ipsilateral & - & - & $3(16.6)$ \\
\hline Bilateral & - & - & $1(5.5)$ \\
\hline
\end{tabular}

OP: ovarian pathology; POC: physiologic ovarian changes; PTC: paratubal cysts 
period has not been reported previously 5 . It is well known that maternal, placental and fetal FSH, LH and estrogen with placental hCG can cause ovarian cysts by stimulating ovarian follicles ${ }^{8}$. Because PTCs are the remnants of tubal (paramesonephric), mesothelial and mesonephric structures, we might speculate that they also respond to the aforementioned hormonal activities during the prenatal period in our three neonatal cases.

Of the 26 PTC cases, 22 of them occurred with a non-neoplastic or neoplastic OP or with a POC. The most frequent histopathologic diagnosis was a simple serous cyst, followed by a corpus luteum cyst (Table I). Simple serous cysts can elevate estrogen levels, and corpus luteum cysts can occur under progesterone influence ${ }^{9}$. Thus, we might also speculate that the occurrence of PTCs with benign OP and POC reflects a possible hormonal mechanism in PTC occurrence.

Only four (15.4\%) solitary PTC cases were identified among the 26 cases in our study, and $84.6 \%$ of the PTCs occurred with an OP or POC. Although the latter finding suggests there is an association between PTCs and OP or POC, it is not clear whether this observation was a coincidence. In a recent report, an association between PCOS and PTCs was mentioned but not clearly demonstrated ${ }^{6}$. To the best of our knowledge, there have been no previous reports speculating an association between ovarian tumors or POC and PTCs, especially in neonates.

Ultrasonography can identify PTCs. Characteristic findings on US are unilocular, anechoic or hypoechoic cysts that stand separate from the ovary 5,10 . When a PTC undergoes torsion, however, it is very difficult to diagnose by US7. In our study, none of the symptomatic cases were identified by US before surgery, except for one uncomplicated solitary PTC case. The latter cyst was approximately $10-\mathrm{cm}$ in its wider diameter and intermittent colicky pain was suggestive of adnexal torsion, thus the patient was operated on. In our study, we did not find any specific laboratory values attributable to PTCs.

The treatment of complicated PTCs is excision, either by laparotomy or by laparoscopy ${ }^{11}$. Although excision of silent PTCs has not been recommended by some authors ${ }^{12}$, prophylactic PTC excision is a common practice in the published literature to prevent possible complications ${ }^{3,11}$. These complications include hemorrhage, perforation, tubal-ovarian torsion, recurrence of torsion and ectopic pregnancy $1,3,13$. Furthermore, it has been reported that paratubal cysts can transform into malignancies ${ }^{4,5,14}$. Tubal-ovarian torsion was treated with salpingectomy in our cases, except in one case. The high tubal-ovarian torsion rate caused by PTCs in our study and the rate up to $45 \%$ in the literature ${ }^{4}$ suggests that prophylactic excision is beneficial before complications occur.

The main drawback of this study was its retrospective nature. Because we did not expect to find PTCs in a majority of the cases, we did not evaluate specific hormones, such as androgens or progestins.

In conclusion, PTCs can occur at any age during childhood, even in the newborn period. Their association with ovarian pathologies or physiologic ovarian process in childhood cases needs to be clarified to further understand the hormonal mechanisms that are influencing the occurrence of these cysts.

\section{REFERENCES}

1. Samaha M, Woodruff JD. Paratubal cysts: frequency, histogenesis, and associated clinical features. Obstet Gynecol 1985; 65: 691-694.

2. Dietrich JE, Heard MJ, Edwards C. Uteroovarian ligament torsion of the due to a paratubal cyst. J Pediatr Adolesc Gynecol 2005; 18: 125-127.

3. Okada T, Yoshida H, Matsunaga T, et al. Paraovarian cyst with torsion in children. J Pediatr Surg 2002; 37: 937-940.

4. Kiseli M, Caglar GS, Cengiz SD, Karadag D, Y1lmaz MB. Clinical diagnosis and complications of paratubal cysts: review of the literature and report of uncommon presentations. Arch Gynecol Obstet 2012; 285: 15631569.

5. Muolokwu E, Sanchez J, Bercaw JL, et al. The incidence and surgical management of paratubal cysts in a pediatric and adolescent population. J Pediatr Surg 2011;46: 2161-2163.

6. Muolokwu E, Sanchez J, Bercaw JL, et al. Paratubal cysts, obesity, and hyperandrogenism. J Pediatr Surg 2011; 46: 2164-2167.

7. Said MR, Bamigboye V. Twisted paraovarian cyst in a young girl. J Obstet Gynaecol 2008; 28: 549-550.

8. Brandt ML, Helmrath MA. Ovarian cyst in infants and children. Semin Pediatr Surg 2005; 14: 78-85. 
9. Von Allmen D, Fallat ME: Ovarian tumors, in Coran AG (ed). Pediatric Surgery. Philadelphia: Elsevier Saunders, 2012: 529-548.

10. Low SC, Ong CL, Lam SL, Beh ST. Paratubal cyst complicated by tubo-ovarian torsion: computed tomography features. Australas Radiol 2005; 49: 136139.

11. Thakore SS, Chun MJ, Fitzpatrick K. Recurrent ovarian torsion due to paratubal cysts in an adolescent female. J Pediatr Adolesc Gynecol 2012; 25: e85-e87.
12. Muthucumaru M, Yahya Z, Ferguson P, Cheng W. Torsion of hydatids of Morgagni in premenarchal adolescent girls--a case report and review of literature. J Pediatr Surg 2011; 46: e13-e5.

13. Seshadri S, Morris A, Uchil D, Joloaso A. Bilatera paratubal cysts with co-existent fallopian tube torsion in an adolescent. J Obstet Gynaecol 2009; 29: 564-565.

14. Lee S, Ahn KH, Park HT, et al. Paratubal Borderline Malignancy: ACase of a 17-Year-Old Adolescent Female Treated with Laparo-Endoscopic Single-Site Surgery and a Review of the Literature. J Pediatr Adolesc Gynecol 2016; 29: 74-76. 